\title{
OnTraJaCS: Ontology based Traffic Jam Control System
}

\author{
Mohamed H. Haggag \\ Computer Science Department \\ Faculty of Computers \& Information \\ Helwan University, Egypt
}

\author{
Doaa R. Mahmoud \\ Computer ScienceDepartment \\ Faculty of Computers \& Information \\ Helwan University, Egypt
}

\begin{abstract}
Traffic jams and congestions have bad effects on drivers and the whole community. Most - if not all - route planning systems focus on finding the optimal/fastest route for its users. Users might be advised to pass through a congested road section if it will take less time to traverse than its alternatives. This approach ignores the negative effects of congestion on both of the individual and the whole community. Instead of focusing on giving each user the fastest route to his destination, this papers aims to develop a model that would be optimal to the whole community. The ultimate objective of the OnTraJaCS is to give all registered vehicles route recommendations that would minimize congestions when they are executed simultaneously. This is achieved by minimizing congestion over the whole map even if some trips will take longer path in order to avoid congestion formation. OnTraJaCS uses Web Ontology to detect and predict congestion and it uses Java to simulate and evaluate possible plans. Simulation results proved that the OnTraJaCS is able to find optimal plans in accordance with the desired criteria and hence fulfilling its objective, with a slight increase in average trip time.
\end{abstract}

\section{Keywords}

Route Planning, Optimization, Congestion avoidance, Traffic Jam, Road Network, Ontology.

\section{INTRODUCTION}

Traffic jams due to congestions that happens at peak hours are characterized by a large increase in the average vehicle trip time (delay). This delay has a negative effect on the growth of economy and trade, fuel usage, vehicles wear and tear, drivers' comfort, driving safety and air purity.

The most important factor that causes traffic jam formulation is traffic density which is number of vehicles per kilometer per lane[1]. Other factors that cause traffic jam too. Some of those factors are temporary, some are permanent and some are periodic. For example, a traffic jam caused by an accident is temporary while a traffic jam caused by a narrow road section is permanent and a traffic jam in front of a theatre is periodic.

The concept of "Semantic Web" was first envisioned by Berners-Lee, Hendler, and Lassila. The trio's vision was published in a famous Scientific American magazine article titled "The Semantic Web: A New Form of Web Content That Is Meaningful to Computers Will Unleash a Revolution of New Possibilities"[2]. The main objective of Semantic Web is to make web content easier for machines to un- derstand and process, thus allowing for the use of artificial intelligence techniques to infer knowledge from it.

One of the main concepts of semantic web is ontology. The word ontology comes from the Greek words ontos (being) + logos (word). In computer science, ontologies were adopted in artificial intelligence to facilitate knowledge sharing and reuse[3]. Ontology is defined in[4]as "an artifact, constituted by a specific vocabulary used to describe a certain reality, plus a set of explicit assumptions regarding the intended meaning of the vocabulary." Ontologies are conceptual models that capture and make explicit the vocabulary used in semantic applications.

The aim of this paper is to improve the efficiency of transportation and to mitigate the damages associated with congestion by giving all registered vehicles route suggestions that would avoid congested roads. OnTraJaCSconsiders the congestions that have already taken place at the time of planning (traffic jam detection) and the congestions that are expected to occur by the time the vehicle reaches that segment on its trip (traffic jam prediction). OnTraJaCSemphasizes on avoiding traffic jam even at the possible cost of increased travel time (to a reasonable extent); Possible plans might advance an uncongested route over a congested one, even if the trip time through the uncongested road is slightly more than it is through the congested one.

The advantage of this prioritization to the whole community is evident in terms of decreased pollution, reduced possibility of accidents and decreased emergency response time (since OnTraJaCSkeeps all roads uncongested). The advantages to individuals using OnTraJaCSare reduced stress and frustration, reduced fuel usage, reduced wear and tear on vehicles and reduced probability of getting involved in road accidents.

OnTraJaCSWeb Ontology technology to detect and predict traffic jam locations and periods based on a multitude of simple data points collected from custom boards installed in all registered vehicles.

\section{RELATED WOKS}

Traffic jam processing applications are classified into three main categories, Traffic Jam Detection, Traffic Jam Prediction and Route Planning. 


\subsection{Traffic Jam Detection}

Traffic jam detection has been addressed in several papers, each of them adopting a different approach. Wei-Hsun Lee et al. addressed this problem by designing a collaborative real-time traffic information generation and sharing framework for intelligent transportation systems[5]. The framework acts in a wiki-like manner by allowing users (car drivers and passengers) to report traffic events in real time through a simple GUI front end and to view the events reported by other users. The system fuses the data collected from users' reports as well as data collected from heterogeneous external real-time traffic information data sources and internal historical traffic information database to gauge the traffic state in real-time.

Jae-Bong Yoo et al. designed a system called INTRACS to address the same problem[6]. INTRACS, short for "Intelligent traffic control system," tracks vehicles that are equipped with RFID tags using RFID readers installed on roadsides. The board containing the RFID readers also contains a few other sensors for temperature, humidity and other factors that affect driving conditions. The board send the info it collects from the RFID reader and sensors to a central server that analyzes it to detect accidents and congestions.

Real-time traffic state detection is also a major part of the cloud-based system designed by Jing Yuan et al.[7]. The system takes advantage of GPS-equipped taxicabs as mobile sensors to probe the city's traffic rhythm constantly and in real-time.

Another system that addressed this problem is "PeerTIS" [8]. The main advantage of PeerTIS is its decentralization. The system uses an overlaid peer-to-peer architecture to take advantage of its decentralized nature without the disadvantages associated with using $\mathrm{p} 2 \mathrm{p}$ in a dynamic and moving network. This works by having all peers' communications routed through the internet. In PeerTIS each vehicle shares its location and speed with its nearest neighbors (geographically). Each peer then analyzes the information received from its neighborhood and calculates traffic state locally.

\subsection{Traffic Jam Prediction}

Traffic state prediction has been addressed in a few papers at different levels of granularity, with some papers relying on micro-level details to predict traffic flow rate at small road segments and others aggregating city-wide statistics to predict traffic flow on macro level.

AndreasRiener and AloisFerscha investigated the effect of individual vehicle parameters on the behavior of the whole group in a specific road segment [9]. The team's simulations have shown that the greatest factor affecting throughput and mean trip time is inter-car distance.

On a slightly higher scale, Takayuki Nakata and Jun-ichi Takeuchi designed a system to predict vehicle travel time over an objective road section using real-time data collected from probe cars [10]. The system collects traffic data from a number of vehicles equipped with sensors and predicts travel time using statistical analysis AR model with seasonal adjustment and MDL (Minimum Description Length) criterion. The system also applies seasonal adjustment to handle periodicities of 24 hours in traffic data.

On larger scale, BogdanTatomir et al. designed a system based on Ant Based Control that is capable of predicting travel time and traffic congestion dynamically [11]. The system uses historic data to predict traffic state on compatible dates (example: on same day of the week), but it also uses real-time collected data to calibrate the estimates in order to accommodate for unexpected events such as accidents.

\subsection{Route Planning}

Hitoshi Kanoh and Kenta Hara modeled route planning as a dynamic multi-objective optimization problem[12]. The three objective functions that need to be optimized simultaneously according to this model are route length, travel time and ease of driving. The system combines hybrid multiobjective genetic algorithm and Dijkstra algorithm to find the Pareto-optimal feasible paths and allows the user to choose a favorite route from the recommended list. 
In addition to its real-time traffic state detection capabilities outlined in section2.1 Traffic Jam Detection, the system proposed by Jing Yuan et al. also offers route planning capabilities. The system relies on historic data, real-time data and driver behavior in order to determine the optimal route that suits its user's driving behavior[7]. The system learns its user's driving behavior gradually from its GPS logs. This info, in addition to real-time data and logs of probe taxicabs and real-time data and logs of weather conditions from internet services are used as input parameters for the system's adaptive algorithm which uses them to find out a user-customized practical fast route for the trip.

Instead of trying to calculate the shortest path based on Euclidian distance or any other criteria, Hector Gonzalez et al. hypothesized that historic data can give more meaningful route recommendations as there must be compelling reasons behind people's decision to frequent specific routes[13]. Such a system takes advantage of human intuition of the masses to find out optimal and practical routes that take into consideration factors that can't be accounted for otherwise. The system uses the hierarchy of roads to partition the road network into areas then uses different path pre-computation strategies at the area level. The system is optimized by considering only routes that are actually travelled frequently in the historic data.

\section{PROPOSED MODEL} ponents:

The proposed model consists of three main com-

1. GIS-to-Ontological Map Representation converter

2. Reasoner and Route Recommendation System (RRRS)

\section{Ontological Logic}

As shown in `Figure 1, digital maps from satellites and other GIS services pass through a conversion process so that they are transformed to OWL files that adhere to the predefined Ontology Map Representation Model. The conversion process is performed once per map.Details about the conversion process and the map ontological representation will be discussed in the next section.

The engine of OnTraJaCSis the Reasoner and Route Recommendation System (RRRS). The RRRS aggregates the data collected from the custom chips installed in all registered vehicles and relates it to the ontology representation of the map receives from the conversion process. Accordingly, the RRRS applies predefined logical rules to the map and collected data in order to device an optimal plan in accordance with the desired parameters.

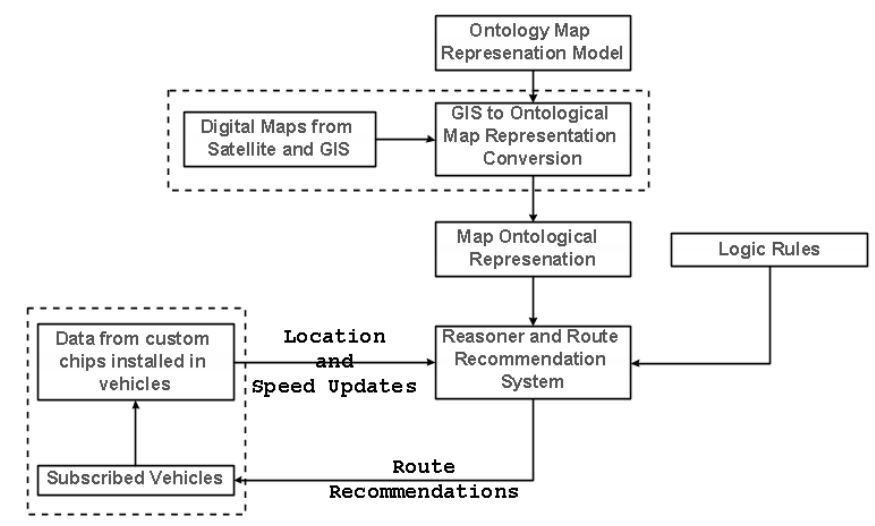

'Figure 1 - OnTraJaCS Components

The RRRS then decimates the suggested plan to all registered vehicles in the form of individual route recommendations. OnTraJaCSaggregates data from the custom chips periodically and re-plans accordingly.

\subsection{Map Ontological Representation}

There are a few general-purpose ontology models for location-based systems available in scientific literature. One of the most comprehensive models is the Domain Ontology of Location Based Systems (LBS) proposed by Dieter Pfoser and NectariaTryfona [14]. This model excels at describing the space/time relations between geographic locations but it doesn't represent traffic flow parameters or temporary events. On the other hand, the data warehouse for traffic control model proposed by LemoniaRagia and Michel Deriaz is well designed to represent traffic flow parameters and temporary events [15].

The two ontology models mentioned above ( [14] and [15]) were utilized and enhanced for use in OnTraJaCS. The traffic control data warehouse ontology model [15] was modified to add the concept of "road segments" whereas a road is represented as a group of road segments instead of being represented as a single road item which intersects with other roads. This makes traffic detection and management more versatile as it allows different road sections to have different parameters. For example: a road section may be narrower than the rest of the road.

Moreover, the Domain Ontology of LBS was simplified by removing some of the classes and features that were not useful in OnTraJaCS'context. This simplification speeds up the system as it reduces the number of entities and relations processed by the reasoner.

A simplified version of the ontology map representation used in OnTraJaCSis shown in Figure 2: 


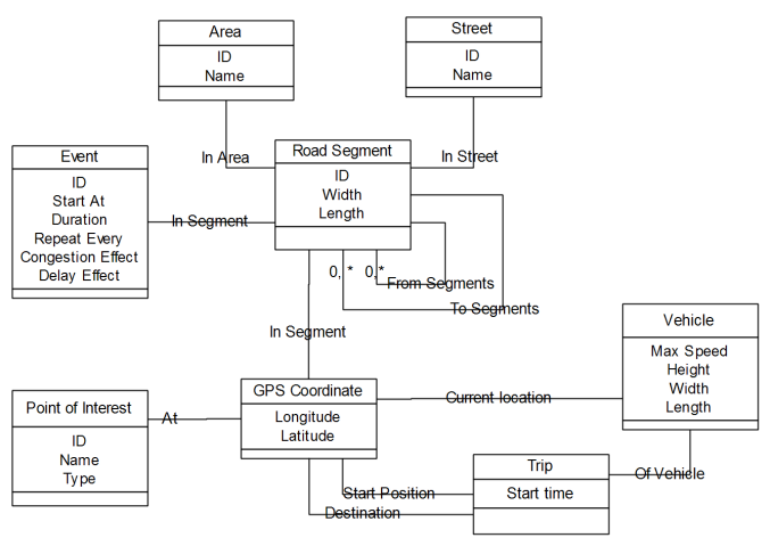

\subsection{Ontological Logic}

The estimates and predictions performed by the ontology play a vital role in the model's accuracy. Ontology's ability to string together simple logic rules and observations to support complex conclusions is leveraged to predict jam-inducing events and stir vehicles away from them.

Offloading all logic-based inference to the ontology has the advantages of simplicity and robustness. In addition, it enablesOnTraJaCSto be adaptable, open and new logic rules can be added and take effect at any time during the model's lifecycle, even while it is deployed and is being actively used.

Following is a sample pseudo code of some of the logical rules employed in OnTraJaCS:

- $\quad$ Segment.Contains(School) $\rightarrow$ Congestion effect at [School.DayStart, School.DayEnd]

- Average Vehicles Speed $<=$ Congestion speed EE Slowdown Period $>=$ Min Threshold E\& Slowdown Period < Max Threshold $\rightarrow$ Segment.Events.Add (Small Accident)

- $\quad$ Average Vehicles Speed <= Congestion speed EE Slowdown Period $>=$ Max Threshold $\rightarrow$ Segment.Events.Add (Permanent Congestion)

- Segment.Contains(Permanent Congestion)
E\& Average Vehicle Speed > Congestion Speed EESpeedUp Period $>=$ Threshold $\rightarrow$ Segment.Events.Remove(Permanent Congestion)

- Segment.Contains(Security Check) $\rightarrow$ Segment.TravelTime $+=$ SecurityCheck.DelayEffect

- $\quad$ Segment.Contains(Theatre) $\rightarrow$ Congestion effect at [Theatre.ShowStart, Theatre.ShowEnd]

- Etc..

\subsection{Reasoner and Route Recommendation System}

The Reasoner and Route Recommendation System (RRRS) acts as the brain that monitors the roads through the data aggregated from the custom chips installed in all registered vehicles.Also it formulates a plan to be followed by the vehicles.

A simple flow chart of the RRRS is shown in Figure 3.

The RRRS starts by categorizing current locations and destinations of registered vehicles into pre-defined geographical categories (neighborhoods/areas). After that, trips that share common source and destination categories are grouped together to minimize the number of units processed. The number of vehicles in each group is stored alongside its source and destination categories.

Next, the RRRS formulates a suitable number of suggested plans. There are a lot ways to achieve this goal; the simplest of which is to generate them randomly. It is preferred however to generate some of the plans systematically based on uniform distribution of percentages or absolute numbers to guarantee that the plans are homogenous.

The task of the RRRS then shifts to simulating and evaluating the available plans then electing an optimal one from them. Each simulation step is performed in two steps. First, the RRRS updates the ontology with the current simulated environment parameters; then the ontology's reasoned applies the predefined logic rules in order to detect congestion and to estimate its effects on various road segments. According to the ontology's reasoner conclusions, the RRRS uses traffic flow theory to move all vehicles another step. 


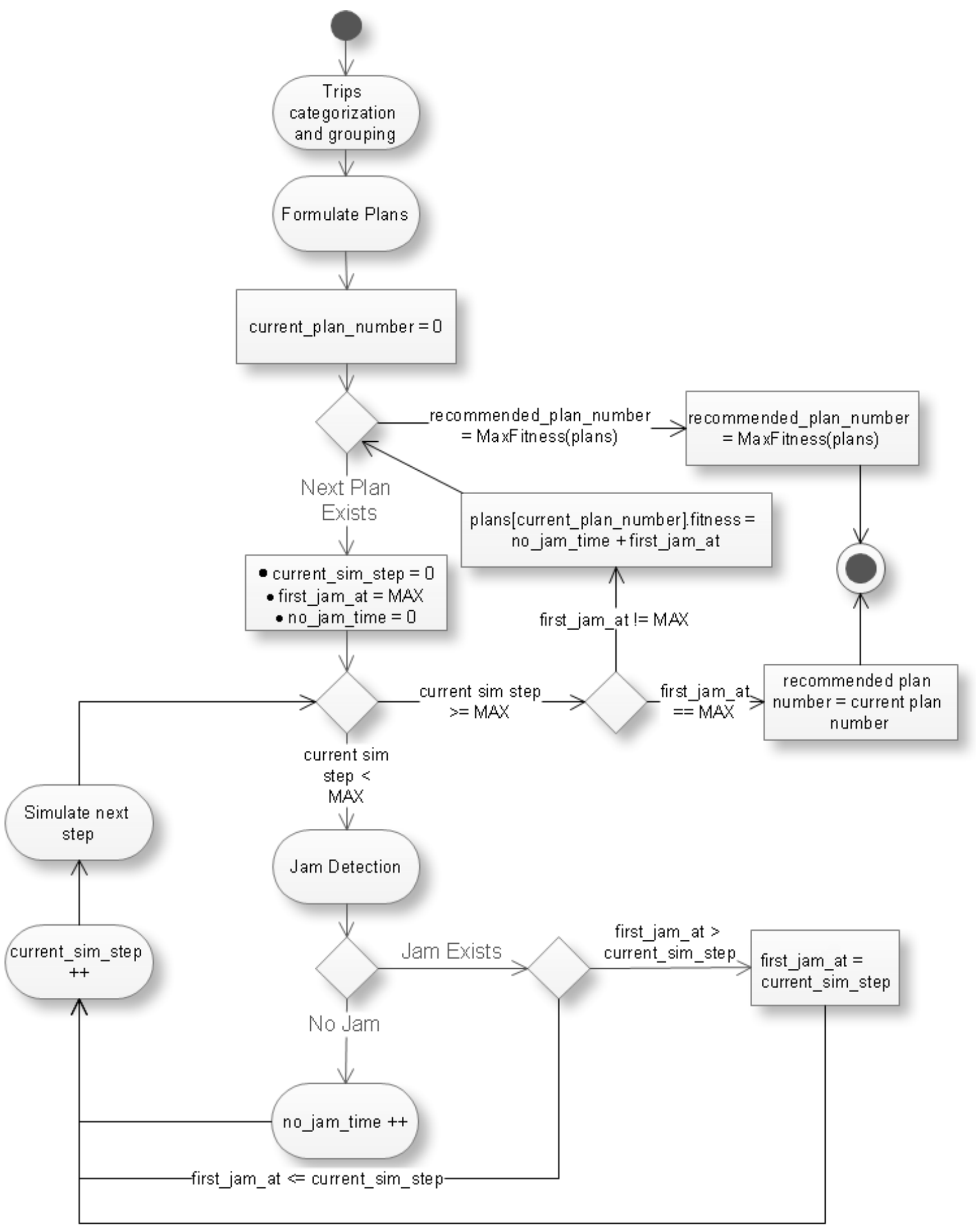

Figure 3 - Processing Activity Diagram 


\subsection{Plans Selection}

Plans are simulated mathematically and the simulation results are compared to find out the optimal plan.

Ideally, the optimal plan should completely avoid congestion throughout the simulation time across all road segments. However, this is impossible in practice, especially when the number of vehicles in the roads exceed the total roads' capacity. Settlement for an optimal plan across all cases is provided.

A good comparison criterion is the length of the time period before the first simulated congestion takes place. This criterion is justified by the fact that simulation accuracy decreases over time. However, this criterion fails if the roads are congested at the beginning of the simulation; in which case, all plans will give zero gain value.

To mitigate this problem another criterion was selected: the total time without congestion throughout the simulation. This criterion isn't affected by the initial state of the model but it is slightly flawed for giving the same credence to simulation predictions at the beginning and end of the simulation interval.

Consequently, both criterions were considered in the final heuristic calculation. The following rules are used to find an ideal or optimal plan:

1. If no congestions occur throughout the duration of the simulation, this plan is ideal. Stop searching and elect it for execution.

2. If no ideal plan is found, plans are compared according to the following heuristic: time before first congestion occurs + total time with no congestion. The plan with highest heuristic value is considered as the optimal plan and is elected for execution.

\section{EXPERIMENT RESULTS}

\subsection{Testing Methodology}

By design, OnTraJaCSis applicable only over large geographical areas that span across different cities (or at least across several districts). The system also requires mildly expensive infrastructure in the form of the central server and the on-board custom electronic chips that should be installed in all vehicles in the area being served. The time, effort and money costs associated with setting up the system for initial use over a large geographical area makes practical real-life experimentation unfeasible and hence simulation was used to test OnTraJaCS.

As stated earlier, the main objective of OnTraJaCSdiffers significantly from that of its most related counterparts. OnTraJaCSaims to minimize roads congestion over the whole map even at the cost of increasing trip times slightly. Most models available both in academia and/or industry aim to minimize trip time for their users regardless of traffic density. A drawback of this discrepancy is that the test results and benchmarks available in literature review is insufficient to make a complete scientific comparison as they only record trip times with no regard to traffic flow densities and congestions. Another factor that increases the comparison difficulty too is that each model is tested on a different map.

One performance measure that has been used in similar situations involves comparing the traffic parameters before and after using the model being investigated. Unfortunately, this is not possible in the case at hand since the since is not deployed in real-life and all testing - in this phase - is simulated. As a result, it was decided that meaningful comparison can be achieved only if the route recommendations of another system were simulated using the same map, traffic parameters and simulation conditions.

Instead of choosing a specific system to benchmark against, OnTraJaCSwas compared to the optimal results obtained by brute force search through all possible routing plans to find the one that satisfies the optimization criteria sought by other systems; i.e. the plan that minimizes average trip time for all subscribed vehicles.

\subsection{Used Map}

OnTraJaCSwas tested on a region in Giza, Egypt. The chosen geographical area spanned across four different districts: Haram/Faisal district, Remaya district, $6^{\text {th }}$ of October district and ShaikhZayed district. The area covered by this region is around $200 \mathrm{Km}^{2}$. This region was chosen due to the availability of required data through several means including satellite maps and field trips.

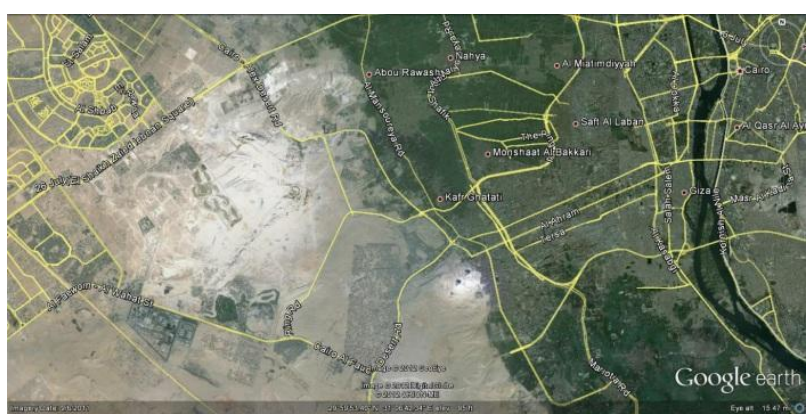

Figure 4: Satellite Terrain View of Selected Region

After trips categorization and grouping, the route recommendations for trips from the Haram/Faisal district to the ShaikhZayed district were evaluated. This group was chosen for evaluation because they are at opposite sides from each other (maximum distance within the application region) and because there is a moderately complex road network connecting them. The directed graph of road segments and intersections connecting the two districts is shown inFigure 5. 


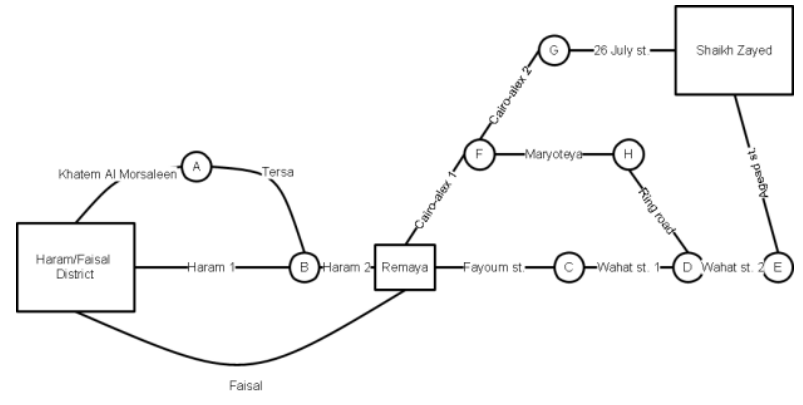

Figure 5: Haram/Faisal District to ShaikhZayed District Directed Graph

Travelers from the Haram/Faisal district can start their journey from any of four main roads in the district: Haram, Faisal, Khatem Al Morsaleen and Tersa. Of those four main roads only Haram street is divided into two road segments in the graph. Needless to say, Haram street comprises tens, if not hundreds, of road segments along its full length of 5.75 kilo meters. However, those real-life road segments are irrelevant to graph since they don't connect main roads. In other words, road segments are defined in the model's context as those that connect the intersections of main roads together.

It is also worth noting that OnTraJaCSleaves room for fine detailed maneuvering off-main roads using other systems. For example a simple weighted shortest path system can be used to find the most suitable side-road to enter Haram Street from TersaStreet and junction "B."

\subsection{Results}

For testing purposes, 30 different scenarios were simulated. The simulation results of the plans recommended by OnTraJaCSwere compared to the optimal results obtained from simulating the basic model's routes which were obtained through brute force search for the same scenarios. The brute force search optimization goal was to minimize the average trip time for all subscribed vehicles on all tracked roads. Brute force search which is guaranteed to find optimal solutions but is illsuited for application in real-time.
Table 1: Brute Forced OptimalAverage Trip Time Plans' Results

\begin{tabular}{|c|ccccc|}
\hline ID & Fitness & $\begin{array}{c}\text { Jam } \\
\text { Count }\end{array}$ & $\begin{array}{c}\text { Average } \\
\text { Trip Time } \\
\text { (minutes) }\end{array}$ & $\begin{array}{c}\mathbf{1}^{\text {st }} \text { Jam } \\
\text { (minutes) }\end{array}$ & $\begin{array}{c}\text { No Jam } \\
\text { Time }\end{array}$ \\
\hline $\mathbf{9 5 6}$ & 128.05 & 2 & 35.55 & 8.05 & 12 \\
$\mathbf{5 9 0}$ & 99.37 & 5 & 69.72 & 9.36 & 9 \\
$\mathbf{1 9 6 2}$ & 128.63 & 2 & 58.68 & 8.625 & 12 \\
$\mathbf{2 7 4 6}$ & 95.75 & 5 & 72.95 & 5.75 & 9 \\
$\mathbf{3 6 6}$ & 115.75 & 3 & 56.86 & 5.75 & 11 \\
$\mathbf{9 8 7}$ & 99.00 & 5 & 60.28 & 9 & 9 \\
$\mathbf{9 5 6}$ & 128.05 & 2 & 35.55 & 8.05 & 12 \\
$\mathbf{2 8 5 8}$ & 130.95 & 2 & 51.44 & 10.94 & 12 \\
$\mathbf{2 2 4 2}$ & 105.12 & 4 & 66.23 & 5.12 & 10 \\
$\mathbf{5 9 0}$ & 115.75 & 3 & 52.24 & 5.75 & 11 \\
$\mathbf{1 9 6 2}$ & 79.42 & 7 & 74.15 & 9.41 & 7 \\
$\mathbf{9 3 7}$ & 108.44 & 4 & 78.56 & 8.44 & 10 \\
$\mathbf{3 9 2 2}$ & 106.53 & 4 & 55.16 & 6.53 & 10 \\
$\mathbf{3 9 2 2}$ & 118.82 & 4 & 84.22 & 18.82 & 10 \\
$\mathbf{2 8 2}$ & 125.75 & 2 & 54.96 & 5.75 & 12 \\
$\mathbf{3 3 3 4}$ & 90.79 & 6 & 71.74 & 10.78 & 8 \\
$\mathbf{7 8 6}$ & 90.00 & 5 & 79.61 & 0 & 9 \\
$\mathbf{2 2 4 2}$ & 104.63 & 4 & 65.97 & 4.62 & 10 \\
$\mathbf{5 9 0}$ & 116.47 & 3 & 57.78 & 6.473 & 11 \\
$\mathbf{3 9 2 2}$ & 88.63 & 6 & 78.31 & 8.625 & 8 \\
$\mathbf{1 7 7 2}$ & 91.59 & 6 & 108.87 & 11.58 & 8 \\
$\mathbf{2 7 4 6}$ & 96.45 & 5 & 66.78 & 6.45 & 9 \\
$\mathbf{7 8 6}$ & 90.00 & 5 & 79.61 & 0 & 9 \\
$\mathbf{3 4 0}$ & 88.79 & 6 & 101.57 & 8.78 & 8 \\
$\mathbf{2 7 4 6}$ & 128.85 & 2 & 45.14 & 8.85 & 12 \\
$\mathbf{1 7 6 6}$ & 119.00 & 3 & 60.49 & 9 & 11 \\
$\mathbf{1 9 8}$ & 127.52 & 2 & 37.03 & 7.52 & 12 \\
$\mathbf{8 0 0}$ & 113.23 & 4 & 59.67 & 13.22 & 10 \\
$\mathbf{3 9 2 2}$ & 83.00 & 6 & 77.95 & 3 & 8 \\
$\mathbf{5 9 0}$ & 99.37 & 5 & 69.72 & 9.36 & 9 \\
\hline
\end{tabular}


Table 2: OnTraJaCS' Results

\begin{tabular}{|c|ccccc|}
\hline ID & Fitness & $\begin{array}{c}\text { Jam } \\
\text { Count }\end{array}$ & $\begin{array}{c}\text { Average } \\
\text { Trip Time } \\
\text { (minutes) }\end{array}$ & $\begin{array}{c}\mathbf{1}^{\text {st }} \text { (minutes) } \\
\text { (mame }\end{array}$ & $\begin{array}{c}\text { No Jam } \\
\text { Time }\end{array}$ \\
\hline $\mathbf{8 6}$ & 135.40 & 2.00 & 48.20 & 48.20 & 15.40 \\
$\mathbf{5 9 0}$ & 99.37 & 5.00 & 69.73 & 69.73 & 9.37 \\
$\mathbf{2 2 1 4}$ & 131.18 & 2.00 & 67.44 & 67.44 & 11.18 \\
$\mathbf{3 0}$ & 105.75 & 4.00 & 78.03 & 78.03 & 5.75 \\
$\mathbf{5 9 0}$ & 117.72 & 3.00 & 57.83 & 57.83 & 7.72 \\
$\mathbf{7 8 6}$ & 119.00 & 3.00 & 62.15 & 62.15 & 9.00 \\
$\mathbf{8 6}$ & 135.40 & 2.00 & 48.20 & 48.20 & 15.40 \\
$\mathbf{3 7 6 8}$ & 149.86 & 3.00 & 58.64 & 58.64 & 39.86 \\
$\mathbf{1 2 3 4}$ & 105.12 & 4.00 & 72.99 & 72.99 & 5.12 \\
$\mathbf{2}$ & 115.75 & 3.00 & 56.44 & 56.44 & 5.75 \\
$\mathbf{8 7 0}$ & 97.82 & 6.00 & 83.20 & 83.20 & 17.82 \\
$\mathbf{2 7 4 6}$ & 112.09 & 5.00 & 89.26 & 89.26 & 22.09 \\
$\mathbf{2 0 4 6}$ & 110.18 & 4.00 & 64.54 & 64.54 & 10.18 \\
$\mathbf{3 9 2 2}$ & 118.82 & 4.00 & 84.23 & 84.23 & 18.82 \\
$\mathbf{8 6}$ & 125.75 & 2.00 & 58.49 & 58.49 & 5.75 \\
$\mathbf{5 9 0}$ & 115.50 & 5.00 & 82.53 & 82.53 & 25.50 \\
$\mathbf{3 1 3 8}$ & 119.43 & 6.00 & 86.60 & 86.60 & 39.43 \\
$\mathbf{1 2 3 4}$ & 104.63 & 4.00 & 71.84 & 71.84 & 4.63 \\
$\mathbf{5 9 0}$ & 116.47 & 3.00 & 57.78 & 57.78 & 6.47 \\
$\mathbf{1 7 6 6}$ & 88.96 & 6.00 & 79.17 & 79.17 & 8.96 \\
$\mathbf{1 7 6 6}$ & 91.59 & 6.00 & 110.79 & 110.79 & 11.59 \\
$\mathbf{5 9 0}$ & 96.62 & 5.00 & 67.14 & 67.14 & 6.62 \\
$\mathbf{3 1 3 8}$ & 119.43 & 6.00 & 86.60 & 86.60 & 39.43 \\
$\mathbf{3 9 2 3}$ & 92.52 & 6.00 & 104.11 & 104.11 & 12.52 \\
$\mathbf{2 1 5 8}$ & 130.29 & 2.00 & 47.39 & 47.39 & 10.29 \\
$\mathbf{9 8 2}$ & 119.00 & 3.00 & 62.58 & 62.58 & 9.00 \\
$\mathbf{7 0 2}$ & 140.49 & 1.00 & 53.81 & 53.81 & 10.49 \\
$\mathbf{3 7 2 6}$ & 122.55 & 6.00 & 69.03 & 69.03 & 42.55 \\
$\mathbf{1 9 6 2}$ & 91.92 & 7.00 & 85.78 & 85.78 & 21.92 \\
$\mathbf{5 9 0}$ & 99.37 & 5.00 & 69.73 & 69.73 & 9.37 \\
\hline & & & & & \\
\hline
\end{tabular}

Basic model's plan ids and their relevant evaluation parameters are listed in table 1. OnTraJaCS' recommended plan ids and their evaluation parameters are listed in table 2. In both tables, the "Plan ID" field refers to its index in the list of all possible routing plan permutations. This list is stored temporarily until a plan is selected for execution, then the other plans are deleted. And even if the plans are not stored (due to memory constraints for example), a given plan index can be converted algorithmically to a complete plan in constant time (time and space complexity $\mathrm{O}(1)$ ). The "fitness" field refers to the heuristic value calculated for the plan (refer to section 3.4 for details regarding the heuristic value calculation). "Jam Count" field counts the absolute number of time a jam occurred at road segment.
The time and magnitude of the jam are not reflected in the "Jam Count." "Average Trip Time" lists the average trip duration for all trips travelled by all vehicles on all roads in the area being investigated. The " 1 st Jam" field relays the total time in minutes that passed after simulation start and until a jam occurred on any road segment. "No Jam Time" is the total time in minutes when there were absolutely no jams in all road segments in the model's application region.

\subsection{Results Discussion}

OnTraJaCS'recommended plans had an average trip time that is $10.2 \%$ higher than those of the basic model, but they kept the roads jam-free for the first 63.35 minutes of the simulated time on average. OnTraJaCS'recommended plans also kept the roads congestion-free for 59.42on average \% more than the basic model's average trip time plans (and up to $338 \%$ better in some cases).

The following graph compares the average trip time for all vehicles when following the routes recommended by OnTraJaCS.

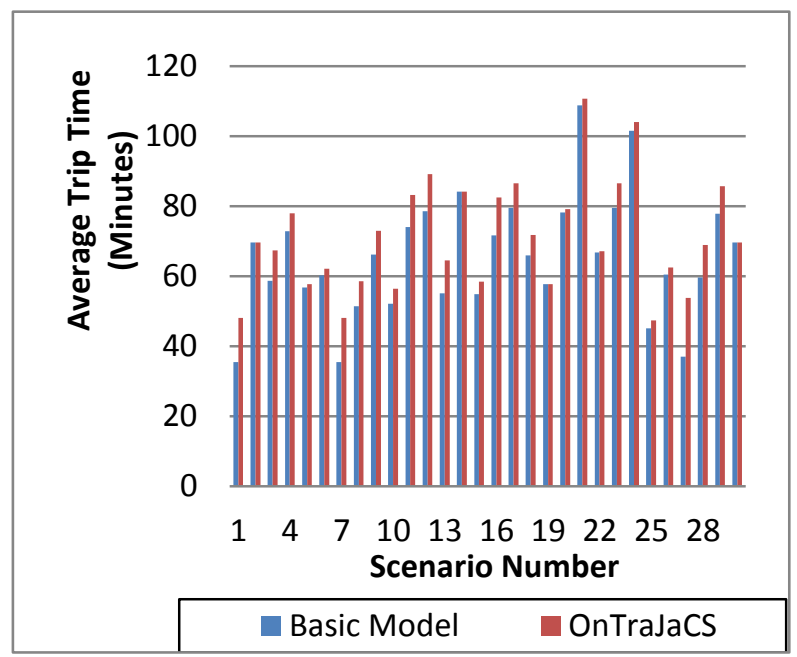

Figure 6: Average Trip Time Comparison

As expected, the graph shows that the average trip time for vehicles that use OnTraJaCSis slightly more than or equal to that of the best possible trip time (exact figures are detailed above).

Similarly, the following graph compares the average time period that passes before the first congestion occurrence on all the roads when following the routes recommended by OnTraJaCS. 


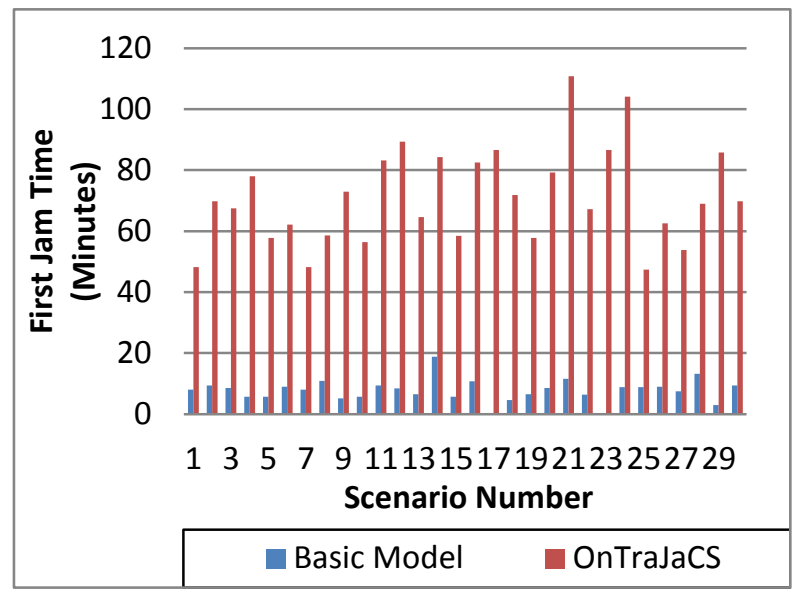

Figure 7: First Jam Delay Comparison

As shown on the graph, when following OnTraJaCS'route recommendations, the first congestion occurred at least after around 50 minutes. The accuracy of any prediction deteriorates with gradually with the passage of time. This means that by pushing congestion as much as possible, the system is highly likely to avoid congestion altogether. This is enforced by the fact that several updates (and re-planning) take place in those 50 minutes before congestion occurs.

Finally, the following graph compares the total jam-free time on all the roads when following the routes recommended by OnTraJaCS.

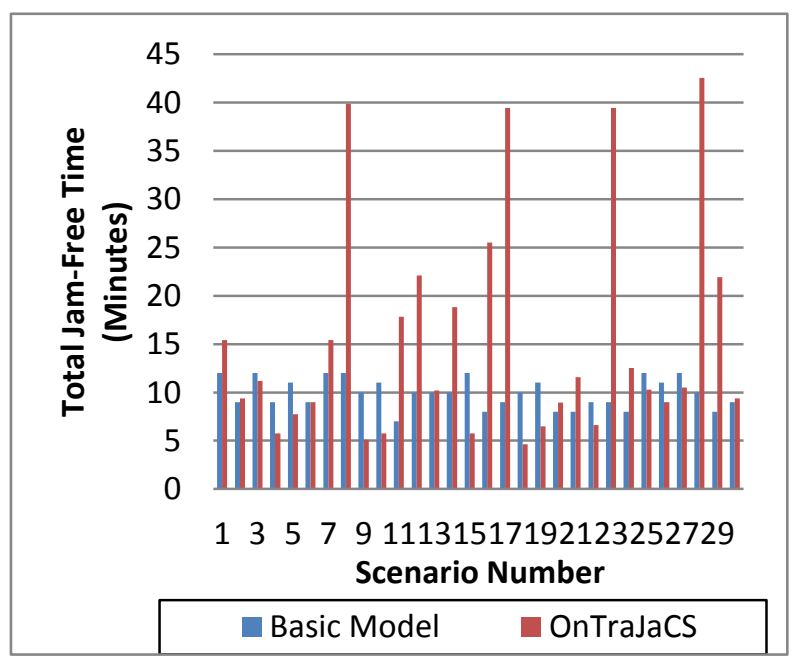

Figure 8: Total Jam-Free Time Comparison

As shown on the graph, OnTraJaCS'recommendations keep the roads congestionfree more than the basic model's average trip time plans for the majority of the cases. However, in some cases OnTraJaCSopts to choose a plan that results in less congestion-free time as long as most of the congestion takes place late enough. This is the desired behavior and its extent can be calibrated empirically by modifying the calibration factor in the Heuristic's equation.

\subsection{Case Study}

To better understand the behavior of OnTraJaCS, a series of experiments were conducted where all conditions were kept constant except the number of vehicles travelling which was incremented by a thousand in each experiment. For simplicity, during this set of experiments all road segments were assumed to be vehicle-free at start. The results of this case study are listed in table 3 .

Table 3: Case Study Results

\section{Results}

OnTraJaCS' selected Traffic Percentage At Each Intersection Exit

\begin{tabular}{rrrrrrrr}
\hline $\begin{array}{c}\text { Vehicles } \\
\text { Count }\end{array}$ & $\begin{array}{c}\text { Jam } \\
\text { count }\end{array}$ & $\begin{array}{c}\text { Average } \\
\text { trip time } \\
\text { (minutes) }\end{array}$ & $\begin{array}{c}\text { Jam-Free } \\
\text { Time } \\
\text { (minutes) }\end{array}$ & & & & \\
KhatemMorsaleen Haram & Faisal \\
\hline 1,000 & 0 & 24.3 & 73.7 & $0 \%$ & $0 \%$ & $100 \%$ \\
\hline 2,000 & 0 & 24.3 & 73.7 & $0 \%$ & $0 \%$ & $100 \%$ \\
\hline 3,000 & 0 & 24.3 & 73.7 & $0 \%$ & $0 \%$ & $100 \%$ \\
\hline 4,000 & 0 & 24.5 & 73.5 & $0 \%$ & $30 \%$ & $70 \%$ \\
\hline 5,000 & 0 & 25.6 & 72.4 & $0 \%$ & $40 \%$ & $60 \%$ \\
\hline 6,000 & 0 & 28.3 & 69.7 & $0 \%$ & $35 \%$ & $65 \%$ \\
\hline 7,000 & 1 & 28.2 & 69.8 & $10 \%$ & $50 \%$ & $40 \%$ \\
\hline 8,000 & 1 & 29.0 & 69.0 & $10 \%$ & $50 \%$ & $40 \%$ \\
\hline 9,000 & 1 & 30.8 & 67.2 & $10 \%$ & $50 \%$ & $40 \%$ \\
\hline 10,000 & 1 & 34.2 & 63.8 & $10 \%$ & $50 \%$ & $40 \%$ \\
\hline 11,000 & 1 & 43.0 & 55.0 & $10 \%$ & $50 \%$ & $40 \%$ \\
\hline 12,000 & 2 & 51.5 & 46.5 & $10 \%$ & $40 \%$ & $50 \%$ \\
\hline 13,000 & 2 & 44.7 & 53.3 & $5 \%$ & $40 \%$ & $55 \%$ \\
\hline 14,000 & 3 & 44.8 & 53.2 & $10 \%$ & $30 \%$ & $60 \%$ \\
\hline 15,000 & 3 & 45.9 & 52.1 & $10 \%$ & $30 \%$ & $60 \%$ \\
\hline
\end{tabular}

\subsection{Case Study Results Analysis}

At the beginning of the experiments, the number of vehicles on map is small enough (Up to 3,000 vehicles on map) to allow OnTraJaCSto divert all vehicles through the single shortest path (Faisal Street - Fayoum Street Wahat Street - Agead Street). Once the number of vehicles on road reaches 4000, the average vehicle speed on Faisal Street falls below the maximum speed limit, leading to an increase in average trip time. OnTraJaCScounters this effect by diverting $30 \%$ of the traffic through Haram Street when the number of vehicles reaches 4,000 .

For on-road vehicles count of 4,000 to 6,000 , OnTraJaCStries to balance the ratio of vehicles traveling through Haram 1 and Faisal road segments to achieve the maximum possible average trip time while avoiding congestion formation in either of them.

Once the number of vehicles on map reaches 7,000 , the first unavoidable congestion occurs. The congestion takes place on the Haram 2 road segment and the only 
way to relieve it is directing more traffic through Faisal Street which is already at its traffic density limit and cannot receive any more vehicles without congesting. OnTraJaCSalso starts diverting $10 \%$ of the traffic through KhatemMorsaleen Street although it is generally slower than Haram and Faisal streets in order to maximize the average speed on the three parallel roads and to prevent congestions formation in Haram and Faisal Streets.

The plan formulated for 7,000 vehicles on road continues to be optimal until number of vehicles reaches 12,000; at which point Haram 1 road segment becomes unavoidably congested and OnTraJaCSstarts increasing the percentage of vehicles that gets diverted through the uncongested alternative road segment, Faisal Street. OnTraJaCSthen keeps balancing the traffic ratios between Haram, Faisal and KhatemMorsaleen streets to maintain minimum possible average trip time while avoiding the formation of any new congestion. This is possible only until the number of vehicles on map reaches 14,000 vehicles. At this point, the number of vehicles on the map is too high and all roads are congested.

\subsection{OnTraJaCS vs. Dijkstra}

The step by step behavior of OnTraJaCSwas compared to Dijkstra's shortest path algorithm. The results of both models are listed side by side in table 4 .

Table 4: Case Study Dijkstra Results

\begin{tabular}{c|ccc}
\hline $\begin{array}{c}\text { Vehicles } \\
\text { Count }\end{array}$ & $\begin{array}{c}\text { Jam } \\
\text { count }\end{array}$ & $\begin{array}{c}\text { Average } \\
\text { trip time }\end{array}$ & $\begin{array}{c}\text { Jam-Free } \\
\text { Time }\end{array}$ \\
\hline 1,000 & 0 & 27.0 & 71.0 \\
\hline 2,000 & 1 & 30.0 & 68.0 \\
3,000 & 1 & 46.1 & 51.9 \\
\hline 4,000 & 3 & 57.4 & 40.6 \\
\hline 5,000 & 4 & 67.0 & 31.0 \\
\hline 6,000 & 4 & 68.1 & 29.9 \\
\hline 7,000 & 4 & 70.5 & 27.5 \\
\hline 8,000 & 4 & 80.2 & 17.8 \\
\hline 9,000 & 5 & 89.6 & 8.4 \\
\hline 10,000 & 5 & 89.6 & 8.4 \\
11,000 & 5 & 89.6 & 8.4 \\
\hline 12,000 & 5 & 89.6 & 8.4 \\
13,000 & 5 & 89.6 & 8.4 \\
14,000 & 5 & 89.6 & 8.4 \\
15,000 & 5 & 89.6 & 8.4 \\
\hline & & & \\
\hline
\end{tabular}

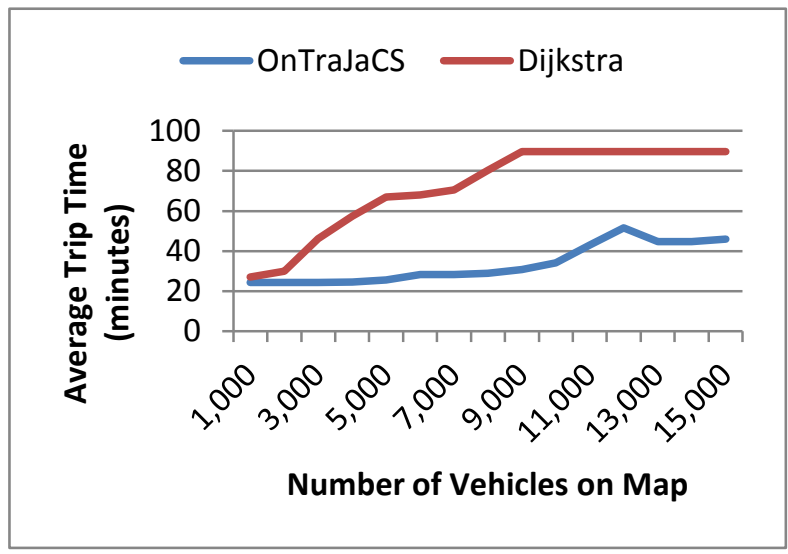

Figure 9: Case Study Results Comparison

Dijkstra algorithm routes all vehicles through the single shortest path route. This leads to the formation of congestion at low traffic density (the first congestion occurred when 2,000 vehicles only were travelling on the map). For medium traffic densities (3,000 to 9,000 vehicles on map), the average trip time of OnTraJaCSincreases at a slower rate than that of Dijkstra. The reason for this is that OnTraJaCSdistributes the vehicles on all available paths intelligently while Dijkstra routes all vehicle through the single shortest path. As the number of vehicles on road reaches 10,000 vehicles, Dijkstra algorithm reaches saturation where all road segments along the shortest path are congested and traffic is flowing at minimal speed. For comparison, OnTraJaCSis able to route 15,000 vehicles at an average trip time of 45.9 minutes while Dijkstra algorithm routes 9,000 vehicles only in 89.6 minutes. It is worth noting that Dijkstra algorithm achieves good results when applied properly according to its design limitations. In practice, systems that use Dijkstra algorithm use it to find shortest path for a relatively small subset of the vehicles on road.

\section{CONCLUSION}

A new model for controlling traffic jam has been provided by this paper. The model is based on Web Ontology. The main objective of OnTraJaCSis to produce route recommendations that minimize or eliminate congestion across its influence area (as opposed to minimizing trip time for certain individuals (subscribers) or overall. The advantages of this approach to the whole community include faster crisis response time, reduced chance of road accidents due to congestion and stress in addition to the economic gains in terms of reduced vehicles depreciation and reduced fuel waste. OnTraJaCSis also advantageous to individual users as it makes their trips stress free, predictable and smoother; not to mention the money they save on car repairs and time lost.

According to the simulation results, OnTraJaCS'recommended plans had an average trip time that is $10.2 \%$ higher than those of the basic model, but they kept the roads jam-free for the first 63.35 minutes of the simulated time on average. OnTraJaCS'recommended plans 
also kept the roads congestion-free for 59.42on average \% more than the basic model's average trip time plans (and up to $338 \%$ better in some cases). Those numbers are in accord with expectations and reflect OnTraJaCS'success in fulfilling its objective. Of course, the tradeoff between average trip time and congestion avoidance is subjective, but it can be reasonably argued that the benefits to the whole community as well as to individuals are worth spending an extra six minutes for every hour of less stressful driving.

\section{REFERENCES}

[1] Boris S. Kerner, Introduction to Modern Traffic Flow Theory and Control: The Long Road to Three-Phase Traffic Theory, 1st ed. Berlin: Springer, 2009.

[2] Tim Berners-Lee, James Hendler, and Ora Lassila, "The semantic web : a new form of web content that is meaningful to computers will unleash a revolution of new possibilities," Scientific American, May 2001.

[3] Dieter Fensel, Ontologies: A Silver Bullet for Knowledge Management and Electronic Commerce, 2nd ed. New York, USA: Springer, 2004.

[4] Nicola Guarino, "Formal ontology in information systems," in proceedings of the first international conference (FOIS'98), Treno, 1998.

[5] Wei-Hsun Lee, Shian-Shyong Tseng, and Wern-Yarng Shieh, "Collaborative real-time traffic information generation and sharing framework for the intelligent transportation system," Information Sciences, pp. 6270, 2010.

[6] Jae-Bong Yoo, Byung-Ki Kim: Ho-Min Jung, ChanYoung Park, and Young-Woong Ko, "INTRACS: Intelligent Traffic Control System Based on Ubiquitous Technology," in INFOS2008, Cairo, 2008, pp. 78-83.
[7] Jing Yuan, Yu Zheng, Xing Xie, and Guang-Zhong Sun, "Driving with Knowledge from the Physical World," in KDD'11, San Diego, 2011, pp. 316-324.

[8] Jedrzej Rybicki, Björn Scheuermann, Markus Koegel, and Martin Mauve, "PeerTIS - A Peer-to-Peer Traffic Information System," in The Sixth ACM International Workshop on VehiculAr Inter-NETworking (VANET 2009), Beijing, 2009, pp. 23-21.

[9] Andreas Riener and Alois Ferscha, "Effect of Proactive Braking on Traffic Flow and Road Throughput," in 13th IEEE/ACM International Symposium on Distributed Simulation and Real Time Applications, Singapore, 2009, pp. 157-164.

[10] Takayuki Nakata and Jun-ichi Takeuchi, "Mining Traffic Data from Probe-Car System for Travel Time Prediction," in KDD'04, Seattle, 2004, pp. 817-822.

[11] Bogdan Tatomir, Leon J.M. Rothkrantz, and Adriana C. Suson, "TRAVEL TIME PREDICTION FOR DYNAMIC ROUTING USING ANT BASED CONTROL," in Winter Simulation Conference, Berlin, 2009, pp. 1069-1078.

[12] Hitoshi Kanoh and Kenta Hara, "Hybrid Genetic Algorithm for Dynamic Multi-objective Route Planning with Predicted Traffic in a Real-World Road Network," in GECCO, Atlanta, 2008.

[13] Hector Gonzalez, Jiawei Han, Xiaolei Li, Margaret Myslinska, and John Paul Sondag, "Adaptive Fastest Path Computation on a Road Network: A Traffic Mining Approach," in VLDB'07, Vienna, 2007, pp. 794-805.

[14] Dieter Pfoser and Nectaria Tryfona, "The Use of Ontologies in Location-based Services:The Space and Time Ontology in Protégé," Research Academic Computer Technology Institute, Athens, Greece,.

[15] Lemonia Ragia and Michel Deriaz, "Location Based Services for Traffic Management," Advanced Systems Group (ASG), Geneva, 2006. 